PROCEEDINGS OF THE

AMERICAN MATHEMATICAL SOCIETY

Volume 111, Number 3, March 1991

\title{
A RESULT BY KULIKOV THAT DOES NOT EXTEND TO MODULES OVER GENERAL VALUATION DOMAINS
}

\author{
L. SALCE AND P. ZANARDO
}

(Communicated by Louis J. Ratliff, Jr.)

\begin{abstract}
Let $R$ be a valuation domain. It is proved that every nonzero $R$-module contains a nonzero pure uniserial submodule if and only if $R$ is rank-one discrete.
\end{abstract}

It is a classical result in the theory of Abelian groups, due to Kulikov [5], that every Abelian $p$-group contains a pure cocyclic subgroup (which is necessarily a summand, cocyclic groups being pure-injective). Note that cocyclic $p$-groups are exactly the torsion uniserial $\mathbb{Z}_{p}$-modules, where $\mathbb{Z}_{p}$ is the rank-one discrete valuation domain obtained by localizing the ring of the integers $\mathbb{Z}$ at the prime $p$.

The goal of this paper is to show that this result is not extendable to valuation domains which are not rank-one discrete. Our main result is the following:

Theorem. Let $R$ be a valuation domain. Then every nonzero $R$-module contains a nonzero pure uniserial submodule if and only if $R$ is rank-one discrete.

The first contribution to the proof of this theorem was given in [2] (see also $[3, \mathrm{X} .4]$ ), where an example of a cohesive $R$-module (i.e., a module without elements of limit height) with no nonzero uniserial pure submodules was given, under the assumption that the maximal ideal $P$ of $R$ is not principal.

The second author showed in [6] that a cohesive $R$-module with the above property cannot exist if and only if $J \cdot R_{J}$ is a principal ideal of $R_{J}$, for each prime ideal $J$ of $R$ (equivalently, $R$ is discrete and $\operatorname{Spec}(R)$ is well ordered by the opposite inclusion); note that the proof of the sufficiency was just an adaptation of the example given in [2].

The consequence of the above results is that, in order to complete the proof of the theorem, we must find a noncohesive $R$-module with no nonzero pure uniserial submodules, where $R$ is any discrete valuation domain with a wellordered prime spectrum consisting of at least two nonzero ideals.

In fact, we shall construct an $R$-module $S$, where $R$ is as in the preceding

Received by the editors October 16, 1989 and, in revised form, March 22, 1990.

1980 Mathematics Subject Classification (1985 Revision). Primary 13C05, 13 C12.

Key words and phrases. Valuation domains, uniserial modules, pure submodules. 
paragraph, which satisfies some particular properties: $S$ has essential socle, all of whose nonzero elements have limit height, and $S$ has a suitable quotient which is a direct sum of cyclics. These properties ensure that our module $S$ has no nonzero pure uniserial submodules.

We will use the following notation. If $R$ is a valuation domain, $Q$ denotes its field of quotients, $P$ its maximal ideal, and $U(R)$ its group of units. For an $R$-module $M$ and an ideal $I$ of $R$, we set $M[I]=\{x \in M: r x=0$ for each $r \in I\}$. For the convenience of the reader, we recall the definition of the height of an element $x \in M$ (see [3, VIII]). The divisibility set of $x$ in $M$ is the subset of $R: D_{M}(x)=\{r \in R: x \in r M\}$; the height ideal of $x$ in $M$ is the submodule of $Q: H_{M}(x)=\cup\left\{R r^{-1}: r \in D_{M}(x)\right\}$. Then, setting $H_{M}(x)=J$, the height of $x$ in $M$ is defined as follows:

$h_{M}(x)= \begin{cases}J / R & \text { if there exists a morphism } \varphi: J \rightarrow M \text { such that } \varphi 1=x, \\ (J / R)^{-} & \text {otherwise. }\end{cases}$

If $h_{M}(x)=J / R$, then we say that $x$ has nonlimit height; otherwise, we say that $x$ has limit height.

An $R$-module $U$ is uniserial if its submodules are linearly ordered by inclusion. A submodule $N$ of an $R$-module $M$ is pure if $r M \cap N=r N$ for all $r \in R$; this is actually the definition of RD-purity, which coincides with Cohn's purity if $R$ is a valuation domain. For all these definitions and for general facts on modules over valuation domains we refer to [3]. Finally, we denote by $\mathbb{N}$ the set of natural numbers, zero included, and by $\mathbb{N}^{+}=\{n \in \mathbb{N}: n \geq 1\}$.

\section{THE MODULE $S$}

Now let $R$ be a valuation domain with principal maximal ideal $P=R p$, such that the prime ideal $P_{1}=\bigcap_{n \in \mathbb{N}} R p^{n}$ is nonzero and principal as $R_{P_{1}}$ module; thus there is an $r \in P_{1}$ such that $P_{1} R_{P_{1}}=r R_{P_{1}}$. Note that $P_{1}$ is generated by the elements $r p^{-n}(n \in \mathbb{N})$.

Let $\Sigma$ denote the set consisting of two symbols $e_{0}$ and $e_{1}$ and the finite sequences of 0 and 1 :

$$
\sum=\left\{e_{0}, e_{1},\left(\sigma_{1}, \sigma_{2}, \ldots, \sigma_{n}\right): n \in \mathbb{N}^{+}, \sigma_{i} \in\{0,1\}\right\} .
$$

Given any $s \in \Sigma$, we define its length $l(s)$ by setting

$$
l(s)= \begin{cases}-1 & \text { if } s=e_{0}, \\ 0 & \text { if } s=e_{1}, \\ n & \text { if } s=\left(\sigma_{1}, \sigma_{2}, \ldots, \sigma_{n}\right) .\end{cases}
$$

For each $s=\left(\sigma_{1}, \sigma_{2}, \ldots, \sigma_{n}\right) \in \Sigma \backslash\left\{e_{0}, e_{1}\right\}$ and different from $(0,0, \ldots, 0)$, let $k(s)=\max \left\{i \leq n: \sigma_{i}=1\right\}$. We define a map $*: \Sigma \backslash\left\{e_{0}\right\} \rightarrow \Sigma$, sending $s$ 
into $s^{*}$, by setting

$$
s^{*}= \begin{cases}e_{0} & \text { if } s=e_{1} \text { or } s=(0,0, \ldots, 0), \\ e_{1} & \text { if } s=(1,0, \ldots, 0) ; \text { i.e., } k(s)=1, \\ \left(\sigma_{1}, \sigma_{2}, \ldots, \sigma_{k-1}\right) & \text { if } s=\left(\sigma_{1}, \sigma_{2}, \ldots, \sigma_{n}\right), \\ & \text { and } k=k(s)>1 .\end{cases}
$$

We remark that $l(s)>l\left(s^{*}\right)$ for all $s \in \Sigma \backslash\left\{e_{0}\right\}$.

Finally, we define the map $t: \Sigma \backslash\left\{e_{0}\right\} \rightarrow \mathbb{N}$ in the following way:

$$
t(s)= \begin{cases}l(s) & \text { if } s=e_{1} \text { or } s=(0,0, \ldots, 0), \\ l(s)-k(s)+1 & \text { otherwise. }\end{cases}
$$

Now let $F=\oplus_{s \in \Sigma} R s$ be the free $R$-module with basis $\Sigma$ and $F_{0}$ the submodule of $F$ generated by the elements

$$
r p e_{0} ; r s-r p^{-t(s)} s^{*}, s \in \Sigma \backslash\left\{e_{0}\right\} .
$$

We shall need the following technical result:

Lemma 1. Each $0 \neq y \in F_{0}$ can be written in the form

$$
y=\sum_{1}^{n} \lambda_{i} s_{i}+r c s
$$

with $0 \neq c \in R, \lambda_{i} \in R, s_{i} \neq s$, and $l(s) \geq l\left(s_{i}\right)$ for each $i$.

Proof. If $y \in \operatorname{Re}_{0}$, then $y=\lambda r p e_{0}$ for $0 \neq \lambda \in R$, so the claim is true. If $y \notin \mathrm{Re}_{0}$, then

$$
y=a e_{0}+\sum_{1}^{m} a_{i}\left(r s_{i}-r p^{-t_{i}} s_{i}^{*}\right)
$$

where $a \in R, 0 \neq a_{i} \in R, s_{i}$ are distinct elements of $\Sigma \backslash\left\{e_{0}\right\}, t_{i}=t\left(s_{i}\right)$ $(i=1, \ldots, m)$. Choose $j \leq m$ such that $l\left(s_{j}\right)$ is maximal. Then obviously $l\left(s_{j}\right)>l\left(s_{i}^{*}\right)$ for all $i \leq m$. Set $s=s_{j}$ and $c=a_{j}$, and the claim follows.

Let $S=F / F_{0}$; for each $s \in \Sigma$, we denote $s+F_{0}$ by $\bar{s}$; then $S$ is generated by the elements $\{\bar{s}: s \in \Sigma\}$, subject to the following conditions:

(2)

$$
\begin{cases}p r \bar{e}_{0}=0 ; r \bar{e}_{0}=r \bar{e}_{1} ; r(\overline{0,0, \ldots, 0})=r p^{-n} \bar{e}_{0} & \text { if } l(0, \ldots, 0)=n ; \\ r(\overline{1,0, \ldots, 0})=r p^{-n} \bar{e}_{1} & \text { if } l(1,0, \ldots, 0)=n ; \\ r \bar{s}=r p^{-t(s)}\left(\overline{\sigma_{1}, \ldots, \sigma_{k-1}}\right)=r p^{-t(s)} \overline{s^{*}} & \text { if } s=\left(\sigma_{1}, \ldots, \sigma_{n}\right) \\ \text { and } k=k(s) \geq 2 . & \end{cases}
$$

From Lemma 1 it follows that $s \neq s^{\prime}$ in $\Sigma$ implies $\bar{s} \neq \bar{s}^{\prime}$ in $S$.

We start by verifying some properties of the elements in $S$.

(P1) For each $s \in \Sigma$ there is an $n(s) \in \mathbb{N}$ such that $r p^{n(s)} \bar{s}=r \bar{e}_{0}$.

We induct on $l(s)$, the claim being trivial for $l(s)=-1$, i.e., if $s=$ $e_{0}$. If $l(s)>-1$, then $r p^{t(s)} \bar{s}=r s^{*}$ and $l\left(s^{*}\right)<l(s)$ gives, by induction, $r p^{t(s)+n\left(s^{*}\right)} \bar{s}=r p^{n\left(s^{*}\right)} \overline{s^{*}}=r \bar{e}_{0}$. 
(P2) For each $x \in S$ there is an $n \in \mathbb{N}$ such that $r p^{n} x=0$; hence $P_{1}^{2} S=0$. It is an immediate consequence of (P1) and relations in (2).

(P3) Every $x \in S$ can be written in the form

$$
x=a \bar{e}_{0}+\sum_{1}^{n} v_{i} p^{n_{i}} \bar{s}_{i}+\sum_{1}^{m} \varepsilon_{j} r p^{-m_{j}} \bar{s}_{j}
$$

where $a \in R$, the $v_{i}$ 's and $\varepsilon_{j}$ 's are either units or zero, $n_{i} \in \mathbb{N}$ for all $i$, and $m_{j} \in \mathbb{N}^{+}$for all $j$.

We can assume $x \notin R \bar{e}_{0}$. First we show that $x$ is of the form

$$
x=a \bar{e}_{0}+\sum_{1}^{m} a_{i} \bar{s}_{i}
$$

with $a \in R, a_{i} \in R$ for all $i$, and $r \in \bigcap_{i} P a_{i}$. We can obviously write $x=b \bar{e}_{0}+\sum_{1}^{m} b_{i} \bar{s}_{i} \quad\left(b, b_{i} \in R\right)$; if $r \in \bigcap_{i} P b_{i}$, then we are done. Let us assume that $b_{j}=c r$ for a $j \leq m(c \in R)$. If $c \in R p^{n\left(s_{j}\right)}$, then $c r \bar{s}_{j} \in R \bar{e}_{0}$ by $(\mathrm{P} 1)$, so $b_{j} \bar{s}_{j}$ can be replaced by $r d \bar{e}_{0}$ for a suitable $d \in R$. If $c=u p^{h}$, with $u \in U(R)$ and $h<n\left(s_{j}\right)$ (this implies that $s_{j} \notin e_{1}$, since $n\left(e_{1}\right)=0$ ), then $r u p^{h} \bar{s}_{j}=r u p^{h} p^{-t\left(s_{j}\right)} \overline{s_{j}^{*}}$. If $h<t\left(s_{j}\right)$, we can replace $r c \bar{s}_{j}=r u p^{h} \bar{s}_{j}$ by $r u p^{h-t\left(s_{j}\right)} \overline{s_{j}^{*}}$, since $r \in \operatorname{Prup}^{h-t\left(s_{j}\right)}$; if $h \geq t\left(s_{j}\right)$, we induct on $h$, since the claim is true for $h=0$. To conclude, it is enough to note that $r \in \bigcap_{i} P a_{i}$ implies that, for each $i \leq m$, either $a_{i}=v_{i} p^{n_{i}}$ for some $v_{i} \in U(R)$ and $n_{i} \in \mathbb{N}$, or $a_{i}=\varepsilon_{i} r p^{-m_{i}}$ for some $\varepsilon_{i} \in U(R)$ and $m_{i} \in \mathbb{N}^{+}$.

(P4) Let $0 \neq x \in S$; then $x \in \bigcup_{k \in N} S\left[p^{k}\right]$ if and only if $x$ can be written in the form

$$
x=r u p^{-h} \bar{e}_{0}+\sum_{1}^{m} r \varepsilon_{j} p^{-m_{j}} \bar{s}_{j}
$$

where $u$ and the $\varepsilon_{j}$ 's are either units or zero, $h \in \mathbb{N}$, and $m_{j} \in \mathbb{N}^{+}$for all $j$.

If $x$ is as in (5), then (P2) ensures that $x \in S\left[p^{k}\right]$ for a suitable $k \in \mathbb{N}$. Conversely, assume that $0 \neq x \in S\left[p^{k}\right]$ for a $k \in \mathbb{N}^{+}$, and write $x$ as in (3). Then, for a suitable $t \geq k, p^{t} \varepsilon_{j} r p^{-m_{j}} \bar{s}_{j}=0$ for all $j \leq m$; so we get

$$
0=p^{t} x=a p^{t} \bar{e}_{0}+\sum_{1}^{n} v_{i} p^{n_{i}+t} \bar{s}_{i}
$$

thus the element of $F: y=a p^{t} e_{0}+\sum_{1}^{n} v_{i} p^{n_{i}+t} s_{i}$ belongs to $F_{0}$. From Lemma 1 we deduce that $v_{i}=0$ for all $i$ and $a=r u p^{-h}$ for a suitable $h \in \mathbb{N}$, and $u$ is a unit of $R$ or zero. Hence $x$ is of the desired form (5).

We consider now some structural properties of $S$. Obviously $S\left[P_{1}\right]=$ $\bigcap_{k \in \mathbb{N}} S\left[r p^{-k}\right]$, and $S\left[P_{1}\right] \supseteq \bigcup_{k \in \mathbb{N}} S\left[p^{k}\right]$. The converse inclusion also holds.

Lemma 2. $S\left[P_{1}\right]=\bigcup_{k \in \mathbb{N}} S\left[p^{k}\right]$. 
Proof. It is enough to prove that, if $x \notin \bigcup_{k \in \mathbb{N}} S\left[p^{k}\right]$, then there exists $t \in \mathbb{N}$ such that $r p^{-t} x \neq 0$. If $x \in R \bar{e}_{0}$, then the claim is obvious, since $R \bar{e}_{0} \cong$ $R / R r p$ [or use Lemma 1 and (P4)]. Assume that $x \notin R \bar{e}_{0}$ and write $x$ as in (3). Then, as in the proof of (P4), one can show that there exists $k \in \mathbb{N}$ such that

$$
p^{k} x=a p^{k} \bar{e}_{0}+\sum_{1}^{n} v_{i} p^{t_{i}} \bar{s}_{i} \quad\left(t_{i} \in \mathbb{N}\right)
$$

where $p^{k} x \neq 0$, since $x \notin S\left[p^{k}\right]$. We show that $x^{\prime}=p^{k} x \notin S\left[P_{1}\right]$, so $x \notin S\left[P_{1}\right]$. Let $t=\max \left\{k, t_{i}: i=1, \ldots, n\right\}+1$; assume that $r p^{-t} x^{\prime}=0$. Then

$$
y=r a p^{k-t} e_{0}+\sum_{1}^{n} v_{i} p^{t_{i}-t} r s_{i} \in F_{0} ;
$$

since $r \in \bigcap_{i} \operatorname{Prp}^{t_{i}-t}$, we deduce from Lemma 1 that $v_{i}=0$ for all $i$, so $x^{\prime} \in R \bar{e}_{0}$; since $x^{\prime} \notin \bigcup_{k} S\left[p^{k}\right], r p^{-h} x^{\prime} \neq 0$ for some $h \in \mathbb{N}$; therefore $x^{\prime} \notin S\left[P_{1}\right]$.

The following lemmas are crucial in proving that $S$ has no nonzero pure uniserial submodules.

Lemma 3. The socle $S[P]$ is essential in $S$.

Proof. Let $0 \neq x \in S$; we must show that $0 \neq a x \in S[P]$ for some $a \in R$. This is obvious if $x \in \bigcup_{k \in \mathbb{N}} S\left[p^{k}\right]$; otherwise, by Lemma 2, there exists $t \in \mathbb{N}$ such that $r p^{-t} x \neq 0$; but $r p^{-t} x \in \bigcup_{k \in \mathbb{N}} S\left[p^{k}\right]$ by (P2), so the claim follows.

Now let $\bar{S}=S / S\left[P_{1}\right]$ and $z_{s}=\bar{s}+S\left[P_{1}\right](s \in \Sigma)$. In this notation we have the following:

Lemma 4. $\bar{S}=\oplus_{s \in \Sigma} R z_{s}$, where $R z_{s} \cong R / P_{1}$ for each $s \in \Sigma$.

Proof. Since $\bar{S}$ is generated by the elements $z_{s}(s \in \Sigma)$, it is enough to prove that $\sum_{0}^{n} \lambda_{i} z_{s_{i}}=0 \quad\left(\lambda_{i} \in R\right)$ implies $\lambda_{i} z_{s_{i}}=0$ for each $i$, or, equivalently, that $\sum_{0}^{n} \lambda_{i} \bar{s}_{i} \in S\left[P_{1}\right]$ implies $\lambda_{i} \bar{s}_{i} \in S\left[P_{1}\right]$ for each $i$. We can assume, without loss of generality, that $s_{0}=e_{0}$. We shall prove that $\lambda_{i} \in P_{1}$ for all $i$; hence the claim will follow from Lemma 2 and (P4). Assume, by way of contradiction, that $\lambda_{1}, \lambda_{2}, \cdots, \lambda_{k} \notin P_{1}$ and $\lambda_{i} \in P_{1}$ for $i \geq k+1 \quad(k \leq n)$. Then $\sum_{k+1}^{n} \lambda_{i} \bar{s}_{i} \in$ $S\left[P_{1}\right]$ and, for $1 \leq i \leq k, \lambda_{i}=u_{i} p^{n_{i}}$ where $u_{i} \in U(R), n_{i} \in \mathbb{N}$. So we get:

$$
\lambda_{0} \bar{e}_{0}+\sum_{1}^{k} u_{i} p^{n_{i}} s_{i}=x \in S\left[P_{1}\right]
$$

If we write $x$ in the form (5) and apply Lemma 1, then we get a contradiction. Thus we have proved that $\lambda_{i} \in P_{1}$ for $i=1, \ldots, n$, from which we derive $\lambda_{0} \bar{e}_{0} \in S\left[P_{1}\right]$. Another application of (P4) and Lemma 1 gives $\lambda_{0} \bar{e}_{0}=\operatorname{rup}^{-h} \bar{e}_{0}$, so also $\lambda_{0} \in P_{1}$. 
We consider now the heights of the elements in the socle of $S$.

Lemma 5. If $x \in S[P]$, then $h_{S}(x) \geq\left(P_{1}^{-2} / R\right)^{-}$.

Proof. Since $P_{1}^{-2}$ is generated by the elements $r^{-1} p^{-n}(n \in \mathbb{N})$, it is enough to prove, looking at (5) in (P4), that for each $s \in \Sigma$ and $h \in \mathbb{N}: r p^{-h_{\bar{s}}} \in$ $\bigcap_{n \in \mathbb{N}} r p^{n} S$. But for each $s \in \Sigma$ there is $s_{0} \in \Sigma$ such that $s=s_{0}^{*}$ and $r \bar{s}_{0}=$ $r p^{-n-h} \overline{s_{0}^{*}} ;$ therefore $r p^{-h} \bar{s}=r p^{-h} \overline{s_{0}^{*}}=r p^{n} \bar{s}_{0} \in r p^{n} S$. Since the choice of $n$ was arbitrary, we are done.

We can now prove the main property of $S$.

\section{Proposition 6. $S$ has no nonzero pure uniserial submodules.}

Proof. Assume, by way of contradiction, that $0 \neq U$ is a pure uniserial submodule of $S$. By Lemma 3, there exists $0 \neq x \in U \cap S[P]$; by Lemma 5 , since $U$ is uniserial pure in $S$ and $P_{1}^{2} S=0$, it follows that $h_{U}(x)=h_{S}(x)=P_{1}^{-2} / R$. Take $y \in U$ such that $r y=x$. Then, by [3, VIII 1.4], $h_{U}(y)=r P_{1}^{-2} / R=$ $P_{1}^{-1} / R$; note that $y \notin S\left[P_{1}\right]$, because of Lemma 2 and the inequalities $p^{k} y \neq 0$ for all $k \in \mathbb{N}$. Hence it follows that the element $0 \neq y+S\left[P_{1}\right]$ of $\bar{S}$ has height $\geq P_{1}^{-1} / R$ in $\bar{S}$, which is obviously absurd, since $\bar{S}$ is a free $R / P_{1}$-module, by Lemma 4.

The proof of our main theorem is now an immediate consequence of Proposition 6. Assume that all the modules over the valuation domain $R$ contain nonzero pure uniserial submodules; then we must prove that $R$ is necessarily rank-one discrete. By Theorem 9 in [6], $R$ is discrete and Spec $R$ is wellordered by the opposite inclusion; so let $P>P_{1}>\cdots$ be the well-ordered sequence of prime ideals of $R$. We must show that $P_{1}=0$. As a matter of fact, if $P_{1} \neq 0$, since $P$ and $P_{1}$ satisfy the hypotheses needed to construct the module $S$, we reach a contradiction by Proposition 6 .

Remark. There are already two available examples of noncohesive modules without nonzero pure uniserial submodules over suitable valuation domains. The first is the divisible module $\partial$, introduced by Fuchs in [1], under the hypothesis that the projective dimension of $Q$ is at least 2 . The second is a weakly polyserial module constructed in [4], which can be obtained, with slight modification, over any valuation domain which fails to be almost maximal. From these two examples one obtains the result that a valuation domain, all of whose modules have nonzero pure uniserial submodules, must satisfy the two additional conditions that Spec $R$ is countable and $R$ is almost maximal. However, it is clear from the above discussion that these results are not useful to prove the theorem in this paper. 


\section{REFERENCES}

1. L. Fuchs, On divisible modules over domains, in Abelian groups and modules, Proceedings of the Udine Conference, CISM Courses and Lectures no. 287, Springer-Verlag, New York and Vienna, 1984, 341-356.

2. L. Fuchs and L. Salce, Uniserial modules over valuation rings, J. Algebra 85 (1983), 14-31.

3. __ Modules over valuation domains, Lecture Notes in Pure Appl. Math., no. 96, Dekker, New York and Basel, 1985.

4. $\ldots$, Polyserial modules over valuation domains, Rend. Sem. Mat. Univ. Padova 80 (1988), 243-264.

5. L. Y. Kulikov, On the theory of Abelian groups of arbitrary cardinality, Math. USSR-Sb. 2 (1941), 165-182. (Russian)

6. P. Zanardo, Valuation domains without pathological modules, J. Algebra 96 (1985), 1-8.

Dipartimento di Matematica Pura e Applicata, Università, Via Belzoni 7, 35131 Padova, Italy

Dipartimento di Matematica Pura e Applicata, Universita dell'Aquila, 67100 L'AQUila, ITALY 